\title{
AN ADVANCED ASTROMETRIC SENSOR ARRAY
}

\author{
David G. Monet and Harold D. Ables \\ U. S. Naval Observatory, Flagstaff Station \\ P.O. Box 1149 \\ Flagstaff, Arizona 86002
}

The U.S.Naval Observatory has commissioned the fabrication of an array of six charge-coupled devices (CCDs) bonded to a single silicon substrate for precision astrometry of sources spanning a large range in apparent brightness. The array includes one CCD with rapid readout capability for imaging bright stars. We present the design parameters and the anticipated performance characteristics of this sensor.

The Electrical Engineering Department of Auburn University under contract to the U.S.Naval Observatory (USNO) has developed the technology to produce an array of six CCDs mounted on a single silicon substrate. Four of these advanced astrometric sensor arrays (AASAs), each composed of 5 Thomson- CSF CCD7882s and 1 Fairchild CCD222, are being fabricated by Auburn University for the USNO. Fabrication of these arrays required development of silicon-on- silicon bonding techniques that survive repeated thermal cycles between $+25^{\circ} \mathrm{C}$ and $-140^{\circ} \mathrm{C}$, electrical isolation of each CCD for independent connections to power and readout electronics subsystems, and accurate geometrical alignment of the CCDs. The AASAs will be used to measure positions of bright stars with respect to much fainter reference fields in "stare" mode operation and to make 3-color photometric observations in "scan" mode operation. A schematic representation of the AASA is shown in Figure 1.

The Fairchild CCD222 is an interline transfer device on which the signal can be electronically shuttered and read out in less than 0.05 second. With typical integration times of 10 minutes on the Thomson-CSF CCDs, the dynamic range of the AASA over which accurate astrometric measurements can be made should exceed 13 magnitudes. Based on experience with a Texas Instruments $800 \times 800$ CCD camera, we expect an astrometric accuracy of \pm 0.005 arcsec for a single observation made under favorable observing conditions and a final accuracy approaching \pm 0.001 arcsec for $\mathbf{4 0}$ or so combined observations. If this performance is realized in practice, absolute parallaxes and proper motions of stars as bright as 4 th or 5 th magnitude can be measured to an accuracy of \pm 0.001 arcsec and the positions of 
these stars can be referred to an inertial extragalactic reference frame (quasi-stellar objects and galaxy nuclei) with the same accuracy.

We anticipate using one of the AASAs in the "scan" mode with appropriately placed color filters for 3-color photometric observations. In this mode the telescope focal plane image on the CCD and the charge image in the CCD are scanned along columns at the same rate. Accurate geometric alignment of the Thomson-CSF CCDs will enable sequential 3-color observations of selected fields. Auburn University employed a chemical milling process to produce a silicon mask for aligning the CCDs to within a few microns during the bonding process.

\section{X) Fairchild 222 Interline Transfer CCD}

(380 columns by 488 rows, $12 \times 18 \mu$ pixels)

\section{+) Thomson-CSF 7882 CCDs}

( 384 columns by 576 rows, $23 \times 23 \mu$ pixels)

(alignment critical for "scan" mode observing)
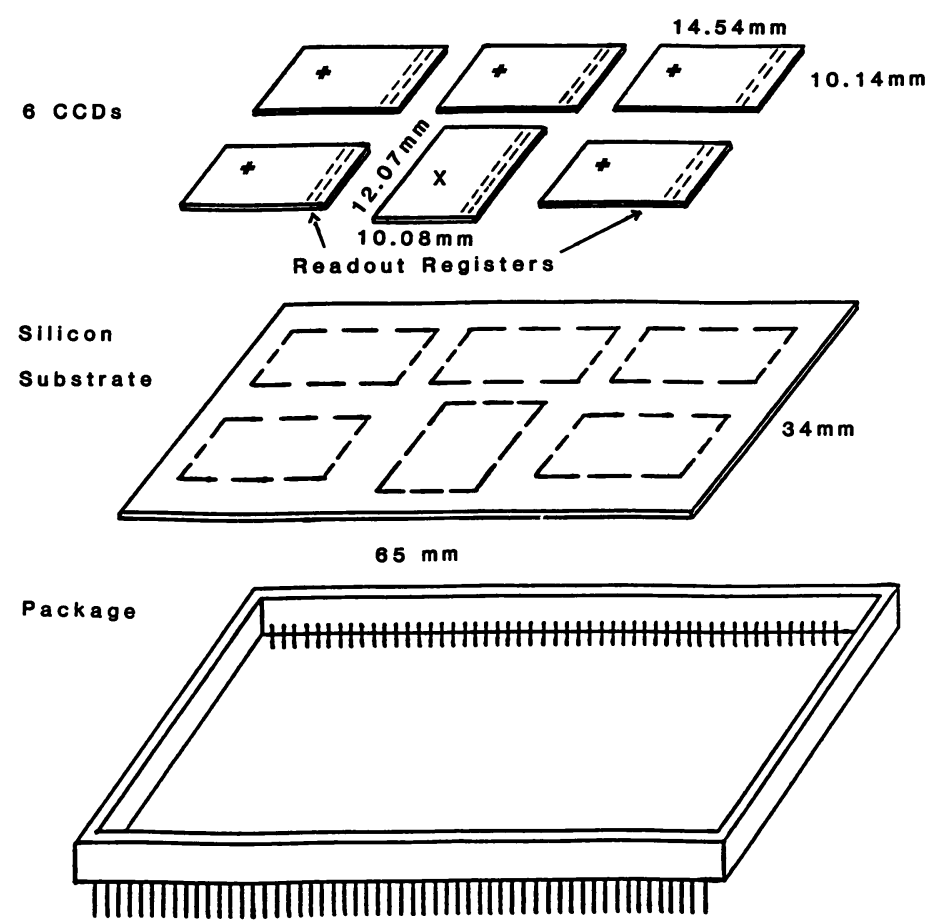

Figure 1. 